\title{
Preparation and characterization of an aromatic polyester/polyaniline composite and its improved counterpart
}

\author{
C. S. $W u^{*}$ \\ Department of Chemical and Biochemical Engineering, Kao Yuan University, Kaohsiung County, Taiwan 82101, \\ Republic of China
}

Received 31 October 2011; accepted in revised form 28 December 2011

\begin{abstract}
Poly(butylene terephthalate) (PBT) composites containing polyaniline (PANI) were prepared using a melt-blending process. Maleic anhydride-grafted PBT (PBT-g-MA) and PANI were used to improve the compatibility of PANI within the PBT matrix. PBT-g-MA/PANI composites exhibited noticeably superior mechanical properties compared with those of PBT/PANI due to greater compatibility with the added PANI. The antibacterial and antistatic properties of the composites were also evaluated. Escherichia coli were chosen as the standard bacteria for determining the antibacterial properties of the composite materials. The PBT-g-MA/PANI composites showed markedly enhanced antibacterial and antistatic properties compared to PBT/PANI composites due to the formation of imide bonds from condensation of the anhydride carboxyl acid groups of PBT-g-MA with the amino groups of PANI. The optimal level of PANI in the composites was $9 \mathrm{wt} \%$, as excess PANI led to separation of the two organic phases, lowering their compatibility.
\end{abstract}

Keywords: polymer composites, poly(butylene terephthalate), polyaniline, antibacterial, antistatic

\section{Introduction}

The revolutionary discovery of metallic properties in molecularly doped polyacetylene opened a new field of research: electronically conducting polymers [1]. Conducting polymers, such as polypyrrole, polyaniline, etc., are polymers with electronically conjugated backbones that, when doped, conduct electricity. These materials have recently attracted much attention due to their potential use in many technological applications that could benefit from their unique polymeric and electronic properties [2-4]. Polyaniline (PANI) is important among known electronic conducting polymers. Several simple chemical methods for synthesis of PANI are known. However, PANI's utilization has been limited because most chemically synthesized PANI is difficult to manipulate using normal melt process- ing techniques. Furthermore, because thin conducting films of PANI are necessary for most applications - such as for electrodes, sensor devices, control of electromagnetic interference, and the dissipation of electrostatic charge - more easily processable PANI-based materials are needed [5-8].

The principal disadvantage of PANI is its poor processability, and for this reason, some of its potential applications have not been fully explored. A reportedly effective method for improving PANI processability is to prepare a core-shell composite using more easily processable polymers for the core material and PANI for the shell material [9-11]. In these kinds of PANI composites, a good balance can be achieved between electrical and mechanical properties. Chen et al. [12] found that core-shell particles of poly(butyl acrylate-methyl methacry-

\footnotetext{
${ }^{*}$ Corresponding author, e-mail: t50008@cc.kyu.edu.tw
}

(c) BME-PT 
late)-polyaniline can be obtained through dispersion of PANI in a bilayer structure of poly(butyl acrylate-methyl methacrylate) latex. Wang and Jing [13] reported a preparation method for polystyrenepolyaniline core-shell structured particles, and they described the properties of their conductive coreshell particles upon incorporation into epoxy-based composites. This study focuses on the effects of functionalized PANI on the electrochemical behavior and conducting properties of poly(butylene terephthalate) (PBT)-based composite materials.

PBT is a thermoplastic, semicrystalline polymer with excellent processability; it has been used widely in structural materials in the electrical, electronics, and automotive industries $[14,15]$. The ongoing practical demands for higher performance materials in various advanced industries has led to the development of new PBT-based composites with additional functional properties, such as high electrical conductivity, antibacterial activity and flame retardance [16-18]. Of these properties, antibacterial activity holds particular interest. The potential benefits of self-sterilizing fabrics made with antibacterial PBT fibers include reduced disease transfer among hospital populations and biowarfare protection. Much research has been conducted to extend and develop commercial applications for PBT in high-performance composites using various reinforcing fillers $[19,20]$.

This study focused on the characterization of composites of PBT-g-MA and PANI prepared using an in situ polycondensation reaction process. To the best of our knowledge, PANI has not yet been evaluated systematically as a reinforcement material in PBT for the production of antibacterial and antistatic composites.

\section{Experimental}

\subsection{Materials}

PBT resins (PBT-1100) were obtained from Chang Chun Corporation (Taiwan). Analytical reagentgrade aniline (purity 99.9\%), ammonium persulfate (SAN, 95\%), ammonium peroxydisulftate (APS, 99.9\%), benzoyl peroxide, and maleic anhydride (MA) were obtained from Sigma-Aldrich Co. (USA). The MA was purified prior to use by recrystallization from chloroform. Benzoyl peroxide was used as a polymerization initiator and was purified by dissolution in chloroform and reprecipitation in methanol. Other reagents were purified using conventional methods.

\subsection{Grafting reaction and grafting percentage}

A mixture of MA and benzoyl peroxide was added in four equal portions at $2 \mathrm{~min}$ intervals to molten PBT to allow grafting to take place and to form MA-grafted PBT (PBT-g-MA). The reactions were performed in a nitrogen atmosphere at $45 \pm 2{ }^{\circ} \mathrm{C}$. Preliminary experiments indicated that the reaction equilibrium was attained in less than $12 \mathrm{~h}$. Thus, reactions were allowed to progress for $12 \mathrm{~h}$ under stirring at a rotor speed of $60 \mathrm{rpm}$. The resultant product $(4 \mathrm{~g})$ was dissolved in $200 \mathrm{~mL}$ of refluxing trifluoroacetic acid/deuterated chloroform $\left(\mathrm{CF}_{3} \mathrm{COOH} / \mathrm{CDCl}_{3}\right)$ solution $(20: 80, \mathrm{v} / \mathrm{v})$ at $40 \pm 2{ }^{\circ} \mathrm{C}$, and the solution was filtered through several layers of cheesecloth. The $\mathrm{CF}_{3} \mathrm{COOH} / \mathrm{CDCl}_{3}-$ soluble product in the filtrate was extracted five times using $600 \mathrm{~mL}$ of cold acetone per extraction. The MA loading of the $\mathrm{CF}_{3} \mathrm{COOH} / \mathrm{CDCl}_{3}$-soluble polymer was determined by titration and expressed as a grafting percentage. Approximately $2 \mathrm{~g}$ of copolymer were heated for $2 \mathrm{~h}$ in $200 \mathrm{~mL}$ of refluxing $\mathrm{CF}_{3} \mathrm{COOH} / \mathrm{CDCl}_{3}$ solution. This solution was then titrated immediately with $0.03 \mathrm{~N}$ ethanolic potassium hydroxide $(\mathrm{KOH})$, which had been standardized against a solution of potassium hydrogen phthalate, with a phenolphthalein indicator. The acid number and the grafting percentage of the PBT- $g$-MA were then calculated using the following Equations (1) and (2) [21]:

Acid number $[\mathrm{mg} \mathrm{KOH} / \mathrm{g}]=$

$$
=\frac{V_{\mathrm{KOH}}[\mathrm{mL}] \cdot C_{\mathrm{KOH}}[\mathrm{N}] \cdot 56.1}{\operatorname{polymer}[\mathrm{g}]}
$$

Grafting percentage $[\%]=$

$$
=\frac{\text { Acid number } \cdot 98.1}{561} \cdot 100 \%
$$

At benzoyl peroxide and MA loadings of 0.3 and $10 \mathrm{wt} \%$, respectively, the grafting percentage was $1.06 \mathrm{wt} \%$.

\subsection{Synthesis of PANI}

The syntheses of PANI and PBT and the preparation of the PBT-PANI composite materials are illus- 


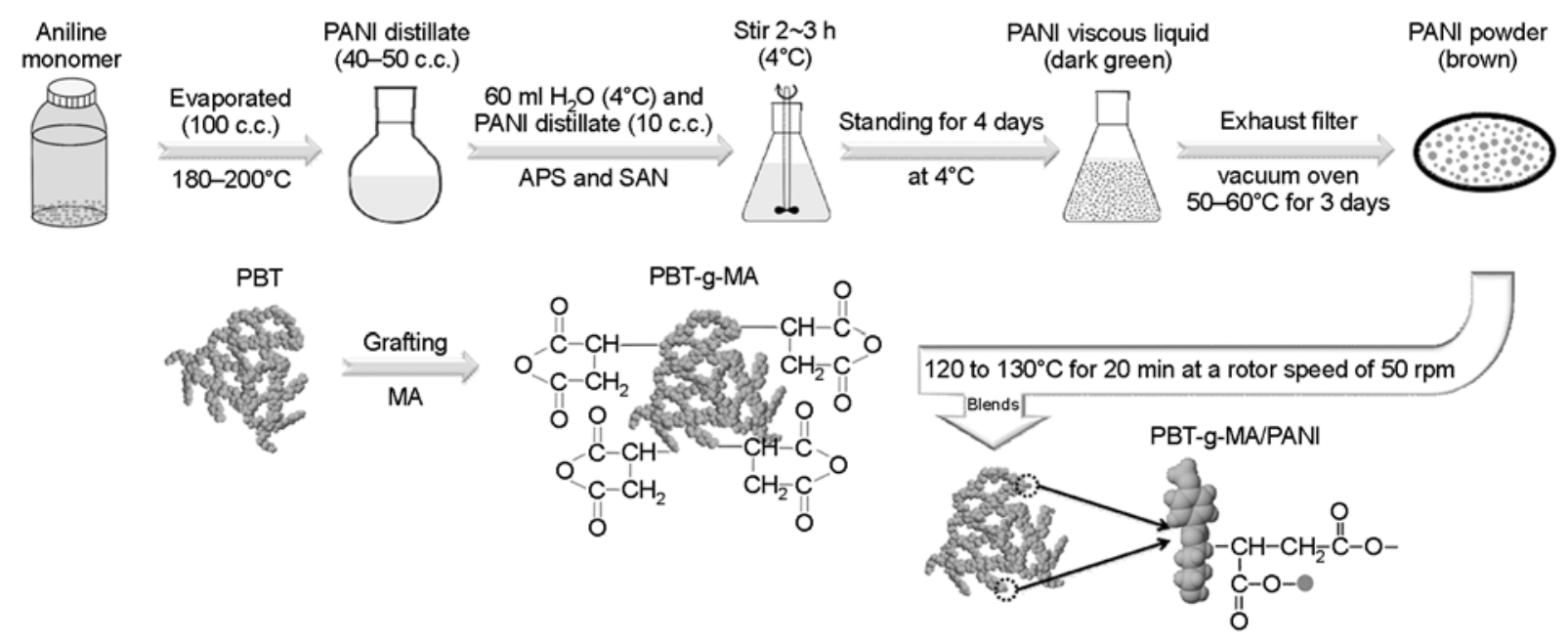

Figure 1. Reaction scheme for the modification of PBT, synthesis of PANI, and the preparation of composite materials

trated in Figure 1. First, a vacuum distillation unit was used to distill and purify $100 \mathrm{~mL}$ of aniline monomer to obtain $40-50 \mathrm{~mL}$ of purified aniline monomer, and then $10 \mathrm{~mL}$ of aniline monomer were drawn by pipette and injected into a conical flask containing $60 \mathrm{~mL}$ of deionized water. Then, $5.74 \mathrm{~g}$ of APS (oxidizer) and $4.76 \mathrm{~g}$ of SAN (monomer) were added to the flask. The flask was placed in an ice bath, the contents were mixed for 2-3 h, and then the flask was placed in a cooling water circulation device and maintained below $4^{\circ} \mathrm{C}$ for 4 days to obtain a green-black viscous liquid. This viscous liquid reaction product was then suction filtered, and the product was washed with deionized water until the wash liquid was clear. The washed product was then placed in a vacuum drying oven, dried at $50-60^{\circ} \mathrm{C}$, and taken out 3 days later. Brown PANI powder was obtained.

\subsection{Preparation of composite materials}

The composites were prepared using a 'Plastograph' $200 \mathrm{Nm}$ W50EHT mixer with a blade-type rotor (C.W. Brabender Instruments, Inc., Hackensack, NJ). Prior to composite formation, PANI samples were dried in a vacuum oven at $50-60^{\circ} \mathrm{C}$ for 2 days. Composites of PBT/PANI or PBT-g-MA/ PANI were prepared with $0,3,6,9,12$, and $15 \mathrm{wt} \%$ PANI filler. Approximately $40 \mathrm{~g}$ of the composite mixture (polymer and filler) were mixed at $50 \mathrm{rpm}$ and $220-230^{\circ} \mathrm{C}$ for $20 \mathrm{~min}$ with an antimony trioxide $\left(\mathrm{Sb}_{2} \mathrm{O}_{3}\right)$ catalyst. Composite mixtures of PBT/ PANI or PBT-g-MA/PANI were placed in a vacuum oven at $70-80^{\circ} \mathrm{C}$ for $24 \mathrm{~h}$. Standard specimens were prepared by pressing the hybrid composites into $1 \mathrm{~mm}$-thick plates using a hydraulic press at $230^{\circ} \mathrm{C}$ and $100 \mathrm{~atm}$. Following pressing, the plates were placed in a dryer for cooling. Prior to characterization, the specimens were conditioned for $24 \mathrm{~h}$ at a relative humidity of $50 \pm 5 \%$.

\subsection{Characterization of hybrid composites}

Fourier transform infrared spectrometry (FTIR; FTS7PC; Bio-Rad, Hercules, CA) was used to investigate the grafting reaction of $\mathrm{MA}$ onto $\mathrm{PBT}$ and to verify ester bond formation between the PANI phase and the PBT matrix. Samples subjected to FTIR analysis were ground into fine powders in a milling machine and pressed into pellets with KBr. Solidstate ${ }^{13} \mathrm{C}$ nuclear magnetic resonance (NMR) analyses were performed with an AMX $400{ }^{13} \mathrm{C}$ NMR spectrometer (Bruker, Madison, WI) at $100 \mathrm{MHz}$. ${ }^{13} \mathrm{C}$ NMR spectra were acquired under cross-polarization and magic angle sample spinning. Power decoupling was employed with a $90^{\circ}$ pulse and $4 \mathrm{~s}$ cycle time. An Instron mechanical tester (Model LLOYD, LR5K type) was used to measure the tensile strength and the elongation at break, in accordance with ASTM D638. Test samples were prepared in a hydrolytic press at $180^{\circ} \mathrm{C}$ and conditioned at $50 \pm 5 \%$ relative humidity for $24 \mathrm{~h}$ before making measurements. Measurements were made using a crosshead speed of $20 \mathrm{~mm} / \mathrm{min}$. Five measurements were performed for each sample, and the results were averaged to obtain a mean value. A thin film of each composite was created using a hydrolytic press and treated with hot water at $80^{\circ} \mathrm{C}$ for $24 \mathrm{~h}$ prior to being coated with gold. The surface morphology of these thin films was observed using 
scanning electron microscopy (SEM; model S-1400, Hitachi Microscopy, Tokyo, Japan). Electrical resistivity was measured directly on laminated films (0.1 mm thick) using an Ohm-Stat RT1000 resistivity meter.

\subsection{Determination of antibacterial properties}

The Escherichia coli (BCRC 10239) were obtained from the Bioresource Collection and Research Center (BCRC; Hsinchu, Taiwan) and maintained in a nutrient broth (NB) medium (3 $\mathrm{g}$ beef extract and $5 \mathrm{~g}$ peptone in $1 \mathrm{~L}$ of distilled water, $\mathrm{pH} 7.0$ ). Nutrient agar was produced by the addition of $15 \mathrm{~g}$ of agar to $1 \mathrm{~L}$ of NB. The bacteria were stored at $-20^{\circ} \mathrm{C}$ in an NB medium containing $60 \%$ glycerol. Prior to testing, $150 \mathrm{~mL}$ of NB medium were inoculated with $10 \mu \mathrm{L}$ of preserved bacteria, and the culture was grown aerobically at $37^{\circ} \mathrm{C}$. After overnight incubation, $3 \mathrm{~mL}$ of the culture were transferred to fresh NB medium and maintained at $37^{\circ} \mathrm{C}$ while shaking at $120 \mathrm{rpm}$ for $18 \mathrm{~h}$. Wells were constructed $(3.00 \mathrm{~cm}$ diameter, $0.05 \mathrm{~cm}$ thick) on the surface of each composite sample $(5.00 \times 5.00 \times$ $0.005 \mathrm{~cm}, 0.05 \mathrm{~cm}$ thick) to hold the bacterial suspension in contact with the sample. A $0.01 \mathrm{~mL}$ aliquot of bacterial suspension $\left(1.26 \cdot 10^{6}\right.$ colonyforming units $[\mathrm{CFUs}] / \mathrm{mL}$ ) was placed on the surface of each sample and covered with a sterile polyethylene film $(4.00 \times 4.00 \times 0.005 \mathrm{~cm}$; Figure 2a) . The samples were incubated at $37 \pm 1^{\circ} \mathrm{C}$ at a relative humidity of $\sim 90 \%$. Following incubation for $24 \mathrm{~h}$, the number of CFUs of E. coli was determined by direct plate counting. Figure $2 b$ shows images of the E. coli colonies from the PBT-g-MA and PBT$g$-MA/PANI ( $9 \mathrm{wt} \%$ ) samples that had been exposed to the composite for various time periods.

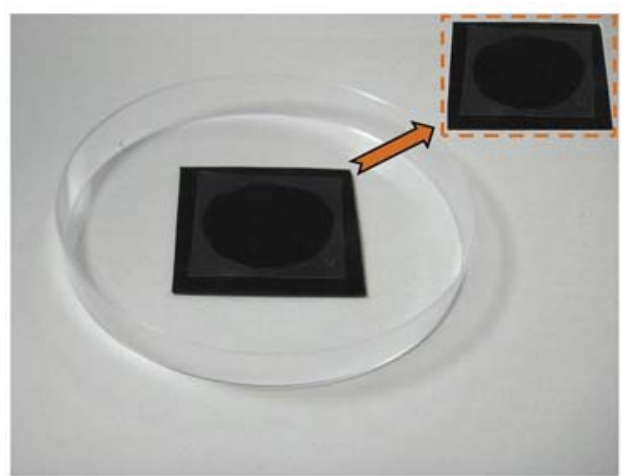

a)
The Japanese Industrial Standard JIS L 1902:1998 is a method typically used to estimate the antibacterial activity of textiles and can also be applied to determine the antibacterial activity of PBT and PANI composites. This method determines the antibacterial index $(\mathrm{ABI})$ and kill-bacterial index $(\mathrm{KBI})$ according to the Equations (3) and (4):

$\mathrm{ABI}=\log B-\log C$

$\mathrm{KBI}=\log A-\log C$

where $A$ represents the number of bacteria that are recovered from the inoculated untreated sample (native PBT or PBT- $g$-MA) immediately following inoculation, $B$ is the number of bacteria remaining in the inoculated untreated sample after $18 \mathrm{~h}$, and $C$ is the number of the bacteria remaining in the inoculated treated sample after $18 \mathrm{~h}$. According to the antibacterial standard of the Japanese Association for the Functional Evaluation of Textiles, an ABI value greater than 2.2 indicates bacterial inhibition, while a KBI greater than 0 indicates a bactericidal effect.

\subsection{Bacteria inhibition tests}

E. coli bacteria were activated in the liquid medium, and the activated bacteria were moved to new medium for $18 \mathrm{~h}$ cultivation. The samples $(100 \mu \mathrm{L})$ were then dropped into the culture dish. Next, a sterilized triangular glass rod was used to spread the bacteria in the dish. In addition, the specimen (about $1.40 \mathrm{~cm}$ diameter, $0.03 \mathrm{~cm}$ thick) was wiped with $75 \%$ alcohol for sterilization and placed into a culture dish with clippers. The culture dish edges were sealed using sealing membrane and placed inside an

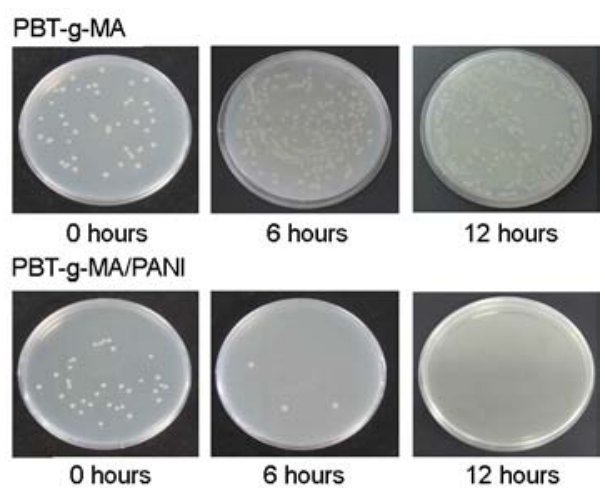

b)

Figure 2. (a) Images of composite samples loaded with a fixed volume $(0.1 \mathrm{~mL})$ of $E$. coli for the evaluation of antibacterial activity. (b) E. coli exposed to PBT-g-MA or PBT-g-MA/PANI (9 wt \%) over time. 
incubator at $30^{\circ} \mathrm{C}$ for $24 \mathrm{~h}$ cultivation to observe the bacterial inhibitory effects.

\section{Results and discussion}

\subsection{FTIR/NMR analyses}

The FTIR spectra of unmodified PBT and of PBT$g$-MA are shown in Figure 3a and 3b, respectively. The characteristic transitions of PBT at 3300 3700, $1700 \sim 1760$, and $500 \sim 1500 \mathrm{~cm}^{-1}$ appeared in the spectra of both polymers [22], with two extra shoulders observed at 1786 and $1857 \mathrm{~cm}^{-1}$ in the modified PBT spectrum. These extra features are characteristic of anhydride carboxyl groups. Similar results have been reported previously [23]. The shoulders represent free acid in the modified polymer and thus denote the successful grafting of MA onto PBT. In the composite PBT/PANI (9 $\mathrm{wt} \%$ ), the $\mathrm{N}-\mathrm{H}$ stretching vibration peak at $3336 \mathrm{~cm}^{-1}$ intensified (Figure 3c) due to contributions from the $-\mathrm{NH}$ group of PANI. The FTIR spectrum of the PBT-g-MA/PANI (9 wt\%) composite in Figure 3d revealed a peak at $1717 \mathrm{~cm}^{-1}$, and a new peak at $3198 \mathrm{~cm}^{-1}$ was observed. This new peak was assigned to the imide stretching vibration of a

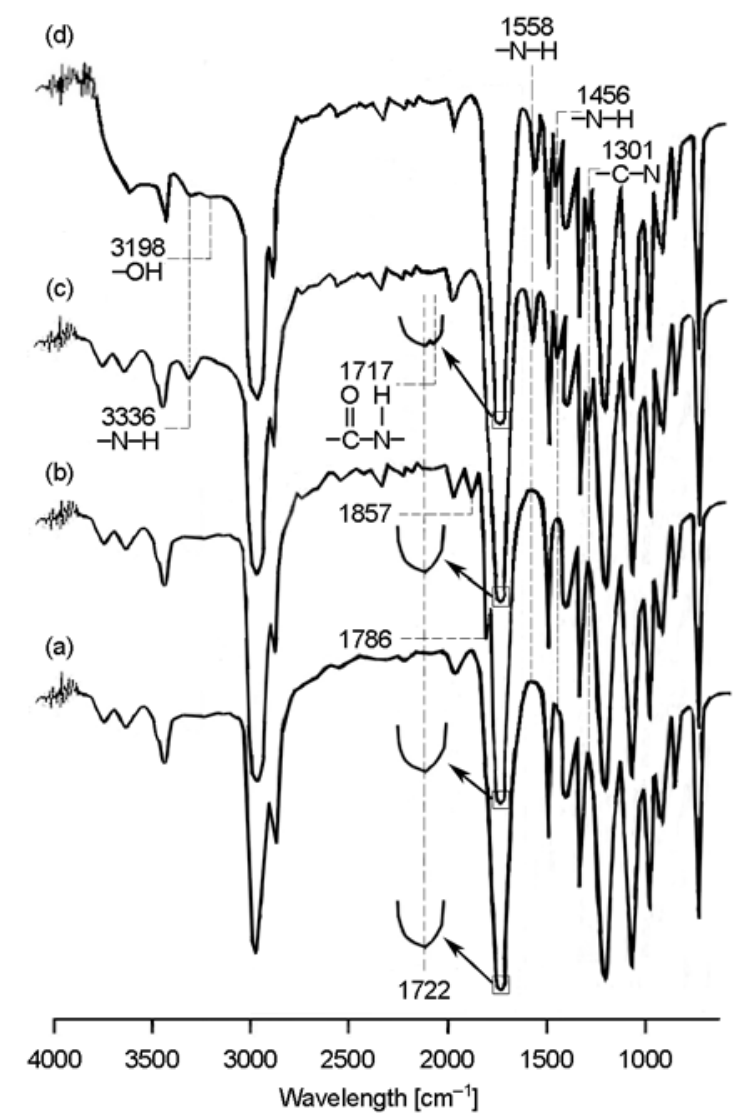

Figure 3. FTIR spectra of (a) PBT, (b) PBT-g-MA, (c) PBT/ PANI (9 wt $\%$ ), and (d) PBT- $g$-MA/PANI (9 wt $\%$ ) copolymer formed via condensation and imide formation between the MA groups of PBT-g-MA and the amino groups of PANI [24]. The characteristic imide stretching vibration peak was not present in the FTIR spectrum of the PBT/PANI ( $9 \mathrm{wt} \%$ ) composite (Figure 3c). Typically, the FTIR spectrum of PANI exhibits peaks in the ranges of 3100 $3500 \mathrm{~cm}^{-1}$ and $1000 \sim 1700 \mathrm{~cm}^{-1}$ [25].

Further evidence for imide formation was provided by ${ }^{13} \mathrm{C}$ NMR spectroscopy. The ${ }^{13} \mathrm{C}$ NMR spectrum of neat PBT (Figure 4a) was similar to that reported by Jansen et al. [26] and showed five peaks: (1) $\delta=$ $170.3 \mathrm{ppm} ;(2) \delta=134.3 \mathrm{ppm}$; (3) $\delta=129.6 \mathrm{ppm}$; (4) $\delta=66.9 \mathrm{ppm}$; and (5) $\delta=27.8 \mathrm{ppm}$. Relative to

(e)
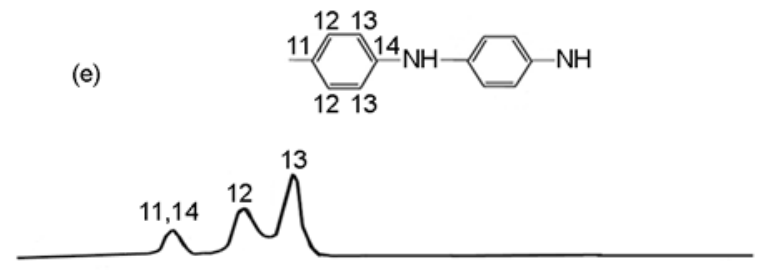

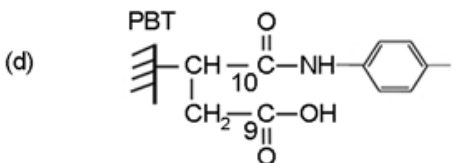
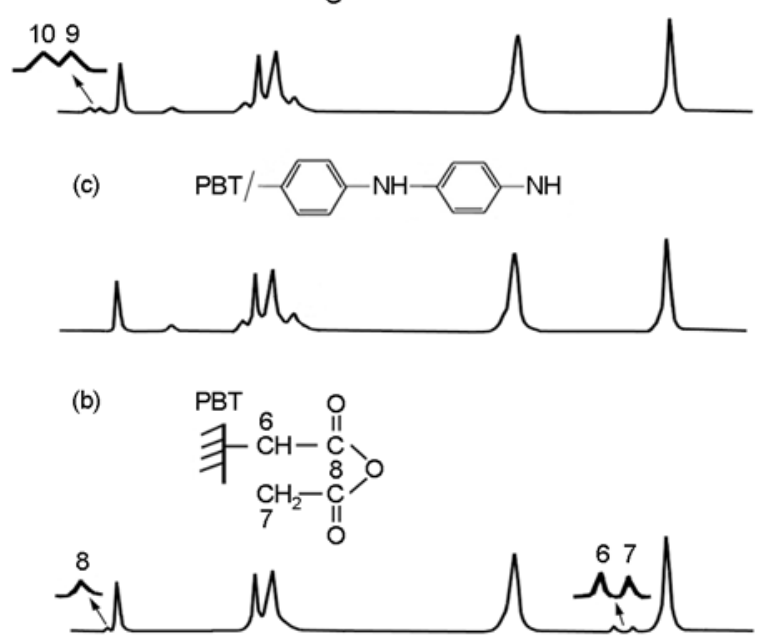

(a) PBT

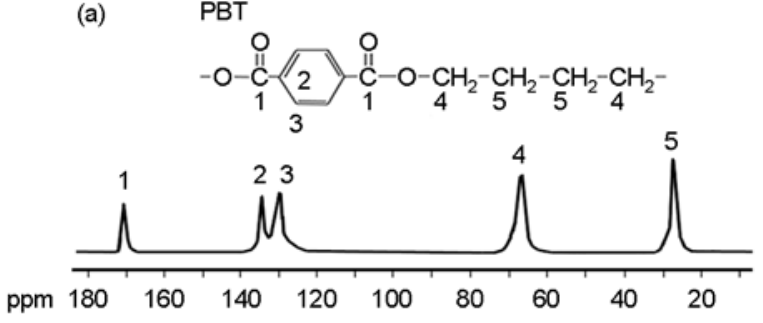

Figure 4. ${ }^{13} \mathrm{C}$ solid-state NMR spectra of (a) PBT, (b) PBT$g$-MA, (c) PBT/PANI (9 wt\%), (d) PBT- $g$-MA/ PANI ( $9 \mathrm{wt} \%$ ), and (e) PANI 
that of neat PBT, the ${ }^{13} \mathrm{C}$ NMR spectrum of PBT- $g$ MA (Figure $4 \mathrm{~b}$ ) contained three additional peaks: (6) $\delta=36.1 \mathrm{ppm},(7) \delta=42.6 \mathrm{ppm}$, and (8) $\delta=$ $172.1 \mathrm{ppm}$. These peaks confirmed the grafting of MA onto PBT. The ${ }^{13} \mathrm{C}$ NMR spectrum of PBT/ PANI, shown in Figure 4c, also exhibited peaks that were not observed in neat PBT and corresponded to aromatic carbon atoms in the PANI ((Figure 4e) (11), (14) $\delta=157.9 \mathrm{ppm} ;(12) \delta=137.8 \mathrm{ppm}$; (13) $\delta=123.2 \mathrm{ppm}$ ). Peaks (9) and (10) (Figure 4d), originating from the reaction between the MA groups of PBT- $g$-MA and the amino groups of PANI, were found at $\delta=175.6 \mathrm{ppm}$ and $\delta=177.1 \mathrm{ppm}$, respectively. These results, combined with the presence of FTIR peaks at 1717 and $3336 \mathrm{~cm}^{-1}$, provide further evidence of imide group formation via condensation of PBT-g-MA with PANI.

\subsection{Hybrid morphology}

The morphology of the PANI and polymer composites can be directly related to their electrical properties. In general, good dispersion of PANI throughout the polymer matrix, effective functionalization of PANI, and strong interfacial adhesion between the two phases is required to obtain a composite material with satisfactory electrical properties. In the current study, PANI may be thought of as a dispersed phase within a PBT or PBT-g-MA matrix. To evaluate the composite morphology, SEM was employed to examine tensile fractures in the surfaces of PBT/PANI ( $9 \mathrm{wt} \%$ ) and PBT-g-MA/PANI (9 $\mathrm{wt} \%$ ) samples. The SEM microphotographs of PBT/PANI (9 wt $\%$ ) in Figure 5a indicated that the PANI in this composite tended to agglomerate into bundles and was unevenly distributed in the matrix. This poor dispersion was due to the formation of hydrogen bonds between PANI and the disparate hydrophilicities of PBT and PANI. Poor wetting in these composites was also noted (Figure 5a) and was attributed to the large difference in the surface energies of the PANI and the polymer matrix [27]. In contrast, the PBT- $g$-MA/PANI ( $9 \mathrm{wt} \%$ ) microphotographs presented in Figure $5 \mathrm{~b}$ indicated a more homogeneous dispersion with improved wetting of PANI in the PBT-g-MA matrix, as indicated by the complete coverage of PBT-g-MA on PANI and the removal of both materials when PANI was pulled from the bulk. This improved interfacial adhesion was attributed to the similar hydrophilicity of the two components, which prevented hydrogen bonding in PANI.

\subsection{Mechanical properties}

Figure 6 shows the variation in tensile strength at break with PANI content for PBT/PANI and PBT$g$-MA/PANI composites. The tensile strength of neat PBT decreased when it was grafted with MA. In PBT/PANI composites, the effect of PANI con-
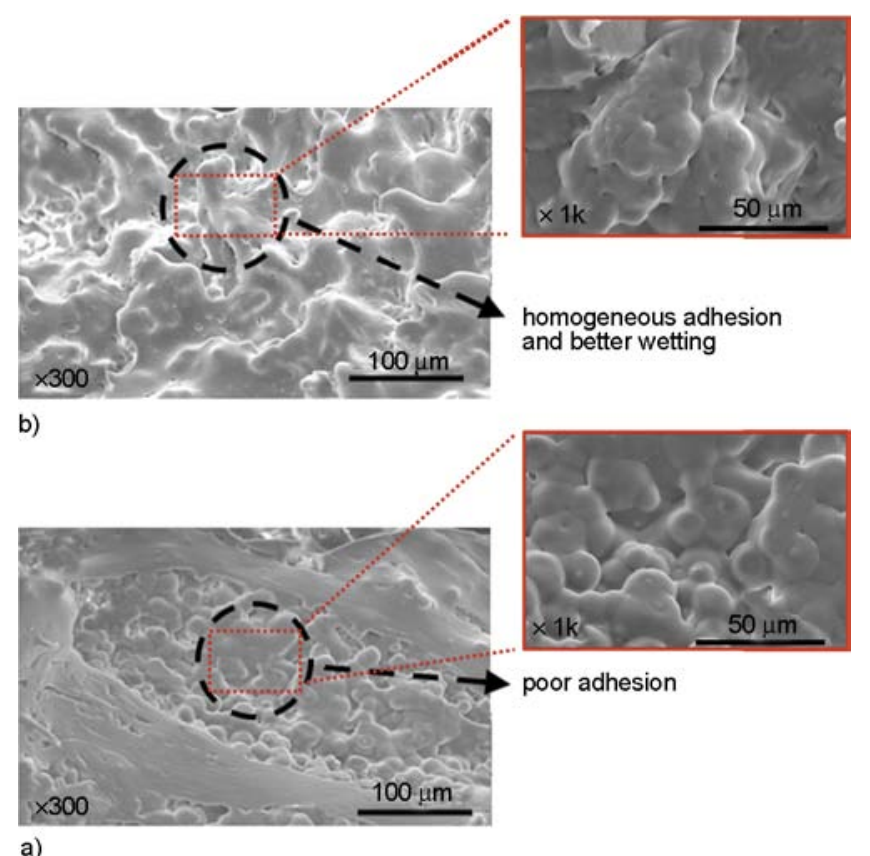

Figure 5. SEM micrographs showing the distribution and adhesion of PANI in (a) PBT/PANI (9 wt\%) and (b) PBT-gMA/PANI (9 wt $\%$ ) composites 


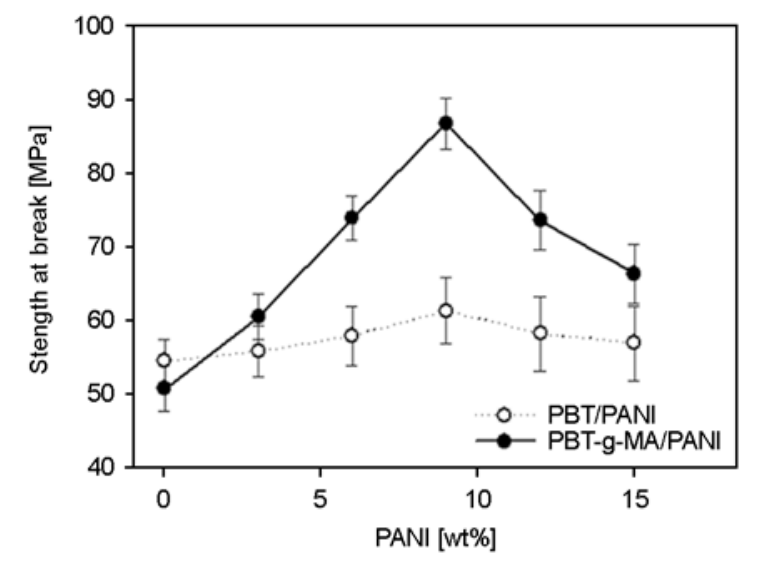

Figure 6. The effect of PANI content on tensile strength at break for PBT/PANI and PBT-g-MA/PANI composites

tent on the tensile strength was somewhat insignificant, which could be attributed to the fact that interfacial forces between the PBT matrix and the PANI consist of only relatively weak hydrogen bonds. In contrast, even though PBT- $g$-MA had a lower tensile strength than pure PBT, the PBT-g-MA/PANI composites exhibited much better tensile strength than the equivalent PBT/PANI composites. In addition, the tensile strength of PBT- $g$-MA/PANI hybrids increased rapidly as PANI content increased from 0 to $9 \mathrm{wt} \%$, at which point the tensile strength approached a plateau and improved only slightly. The positive effect that PANI had on the tensile strength of PBT- $g$-MA was attributed to three factors: (1) the presence of PANI and the consequent formation of chemical bonds through dehydration of MA groups in PBT-g-MA, which immobilized or partially immobilized the polymer phases and added stiffness to the composite layers; (2) the high aspect ratio and surface area of the PANI; and (3) the powder dispersion of PANI layers in the polymer matrix. The data also suggested that PANI layers and molecular orientation contributed to the observed reinforcement effect. The slight decrease in tensile strength observed when the PANI content was above $9 \mathrm{wt} \%$ was attributed to the inevitable aggregation of PANI at higher PANI contents and is similar to the deterioration in other properties observed when PANI content was above $9 \mathrm{wt} \%$. Together, these results support theoretical and molecular simulation predictions that stress transfer, and hence strength, of powder-polymer composites can be effectively increased by the formation of chemical bonds between the components [28-30].

\subsection{Electrical properties}

The electrical conductivities of PBT/PANI and PBT-g-MA/PANI composites were obtained by calculating the reciprocal of measured resistivity values and are summarized in Figure 7. As expected, both PBT/PANI and PBT-g-MA/PANI exhibited lower electrical resistivities than pure PBT and PBT-g-MA. Initially, the electrical resistivities decreased markedly with increasing PANI content, and then they decreased slightly when the content of PANI was greater than $9 \mathrm{wt} \%$. Moreover, the electrical resistivity of each PBT-g-MA/PANI hybrid was lower than that of its PBT/PANI equivalent. The reduced electrical resistivities of PBT-g-MA/ PANI composites compared to those of PBT/PANI composites were attributed to imide bond formation, which inhibited polymer motion in the MAgrafted materials and prohibited the chain rearrangement and reorganization required for solidification. The imide linkages formed in the PBT- $g$-MA/PANI were stronger than the hydrogen bonds formed in PBT/PANI and were thus more effective at hindering polymer motion. In turn, this lower resistivity also allowed the PBT-g-MA/PANI composites to more easily form an interconnected conductive pathway throughout the material. The lower observed resistivity in the $9 \mathrm{wt} \%$ composites indicated that an excess amount of PANI was present at levels $>9 \mathrm{wt} \%$ and that PANI was physically dispersed throughout the polymer matrix. This excess may have led to aggregate formation phases, thereby reducing the matrix compatibility with $\mathrm{PBT} / \mathrm{PANI}$ and PBT-g-MA/PANI.

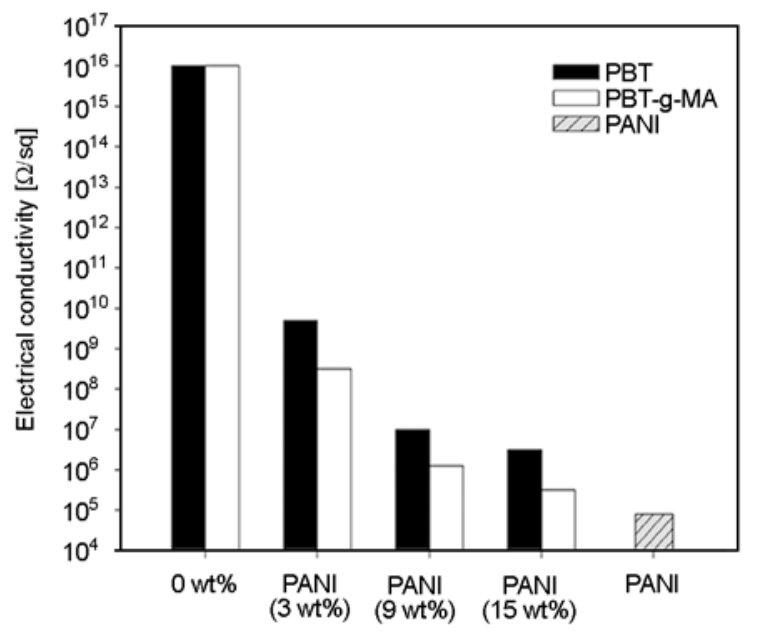

Figure 7. Electrical resistivities of PBT, PBT-g-MA, PBT/ PANI, and PBT- $g$-MA/PANI composites 
Although the $9 \mathrm{wt} \%$ PBT/PANI and PBT-g-MA/ PANI composites exhibited resistivity values as low as $1.1 \cdot 10^{7}$ and $8.1 \cdot 10^{6} \Omega / \mathrm{sq}$, they cannot be classified as conductive materials $[31,32]$. The results of the antistatic testing with pristine PBT- $g$-MA and PBT- $g$-MA/PANI (sample size: $3.50 \mathrm{~cm}$ diameter and $0.10 \mathrm{~cm}$ thick) are depicted in Figure 8. Each film was rubbed and immersed in small plastic foam balls. Any adsorption of the plastic balls indicates the presence of static electricity. The pristine PBT-g-MA adsorbed a significant number of plastic balls, while the $3 \mathrm{wt} \%$ PBT- $g$-MA/PANI composite (Figure 8b) showed only a small number of balls attached to the surface. Furthermore, both the 9 and

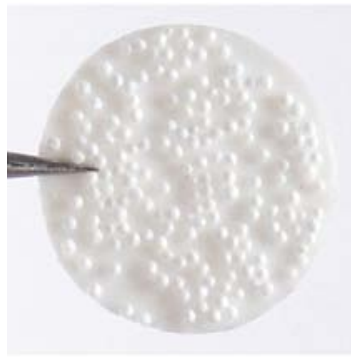

a)

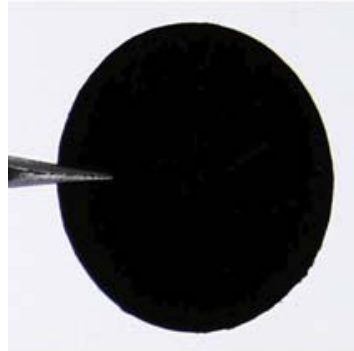

c)

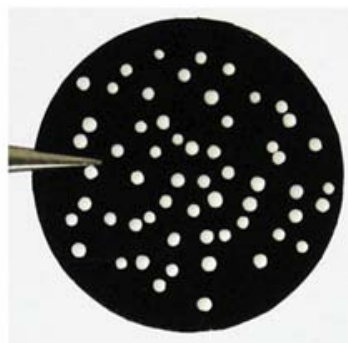

b)

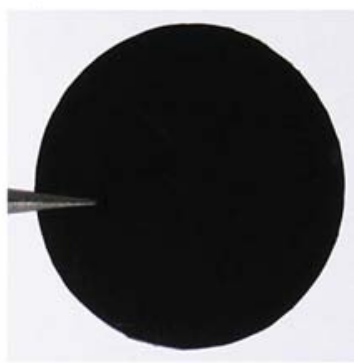

d)
Figure 8. Antistatic properties of (a) PBT- $g$-MA, (b) PBT- $g$ MA/PANI (3 wt \%), (c) PBT-g-MA/PANI (9 wt \%), and (d) PBT-g-MA/PANI (15 wt $\%$ ) composites

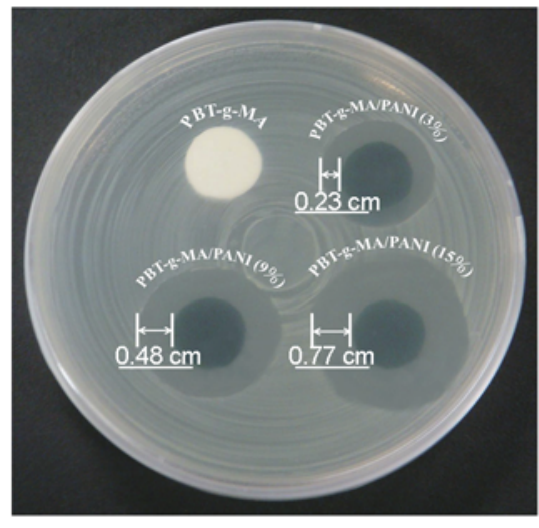

a)
$15 \mathrm{wt} \%$ PBT- $g$-MA/PANI composites (Figure 8c, 8d) were clean due to its lower electrical resistivity $\left(<10^{9} \Omega / \mathrm{sq}\right)$. Although, the electrical resistivity of $3 \mathrm{wt} \%$ PBT-g-MA/PANI $\left(6.7 \cdot 10^{8} \Omega / \mathrm{sq}\right)$ was in the range of dissipative materials, some plastic balls were adsorbed on the surface. The electrostatic dissipative property of the $3 \mathrm{wt} \%$ sample was not sufficient when compared to that of the $9 \mathrm{wt} \%$ $\left(8.1 \cdot 10^{6} \Omega / \mathrm{sq}\right)$ and $15 \mathrm{wt} \%\left(3.4 \cdot 10^{5} \Omega / \mathrm{sq}\right)$ samples. From this result, it can be deduced that the samples exhibit absolute electrostatic dissipation when the electrical resistivity is $<10^{8} \Omega / \mathrm{sq}$. Thus, the addition of PANI to the composite resulted in electrostatic dissipative properties in the bulk material, particularly when the PANI content was greater than $9 \mathrm{wt} \%$.

\subsection{Antibacterial properties of the composites}

Figure 9a illustrates the results of the antibacterial properties of the PBT/PANI and PBT-g-MA/PANI composites. Figure 9a shows that the PBT-g-MA without incorporated PANI displayed no antibacterial zones, indicating that the pure PBT-g-MA materials possessed no antibacterial properties. However, some micro-sized antibacterial zones were found in the PBT-g-MA/PANI composite specimens with as little as $3 \mathrm{wt} \%$ PANI, and with increasing PANI content, the antibacterial zones became more apparent. This indicated that the PBT-g-MA/ PANI composites displayed an antibacterial effect due to the addition of PANI.

Antibacterial activity was evaluated with clinically infectious E. coli As shown in Figure 9b, when in contact with PBT or PBT-g-MA, the E. coli cell count increased with time from $1.26 \cdot 10^{6}$ to

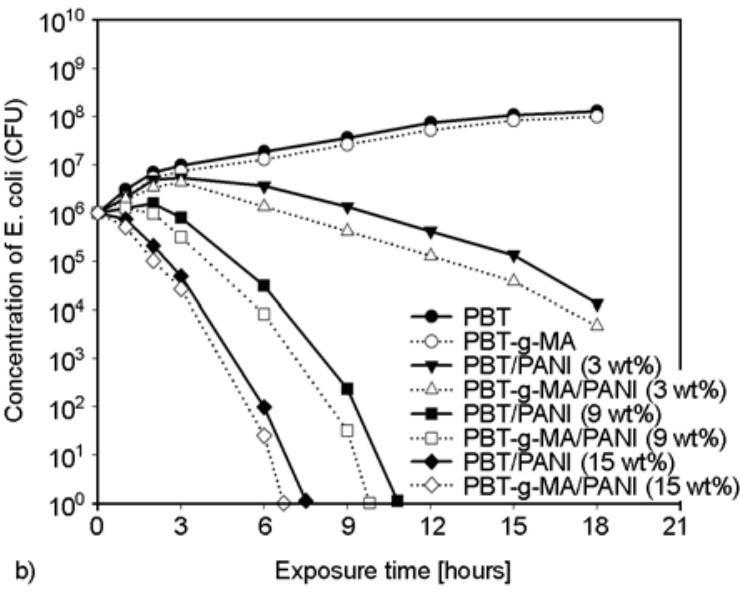

Figure 9. Exposure time course of the (a) growth and (b) inhibition zones of $E$. coli cells during exposure to PBT or PBT$g$-MA and its composite surfaces 
Table 1. Effects of PANI content on the antibacterial properties of PBT/PANI and PBT- $g$-MA/PANI composites

\begin{tabular}{|l|c|c|c|c|c|c|}
\hline & \multicolumn{3}{|c|}{ PBT/PANI } & \multicolumn{3}{c|}{ PBT-g-MA/PANI } \\
\cline { 2 - 8 } & $\mathbf{3} \mathbf{w t} \%$ & $\mathbf{9} \mathbf{w t} \%$ & $\mathbf{1 5} \mathbf{w t} \%$ & $\mathbf{3} \mathbf{w t} \%$ & $\mathbf{9} \mathbf{w t \%}$ & $\mathbf{1 5} \mathbf{w t \%}$ \\
\hline $\mathrm{A}$ & $1.26 \cdot 10^{6}$ & $1.26 \cdot 10^{6}$ & $1.26 \cdot 10^{6}$ & $1.26 \cdot 10^{6}$ & $1.26 \cdot 10^{6}$ & $1.26 \cdot 10^{6}$ \\
\hline B & $1.31 \cdot 10^{8}$ & $1.31 \cdot 10^{8}$ & $1.31 \cdot 10^{8}$ & $1.28 \cdot 10^{8}$ & $1.28 \cdot 10^{8}$ & $1.28 \cdot 10^{8}$ \\
\hline C & $1.33 \cdot 10^{4}$ & 0 & 0 & $6.70 \cdot 10^{3}$ & 0 & 0 \\
\hline ABI & 3.99 & - & - & 4.28 & - & - \\
\hline KBI & 1.98 & - & - & 2.27 & - & - \\
\hline
\end{tabular}

$1.31 \cdot 10^{8} \mathrm{CFU} / \mathrm{mL}$ and from $1.26 \cdot 10^{6}$ to $1.28 \cdot 10^{8} \mathrm{CFU} / \mathrm{mL}$, respectively, after incubation at $37^{\circ} \mathrm{C}$ for $24 \mathrm{~h}$. Conversely, under the same conditions, the bacterial cell count rapidly decreased to near zero when in contact with composites PBT/ PANI or PBT- $g$-MA/PANI containing more than $9 \mathrm{wt} \%$ PANI. At $9 \mathrm{wt} \%$ PANI, the onset of E. coli reduction was observed at $3 \mathrm{~h}$, which was significantly shorter than the approximately $6 \mathrm{~h}$ onset observed with the $3 \mathrm{wt} \%$ PANI composites. This effect is most likely due to the surface density of PANI. As noted in Table 1, according to the guidelines set forth by JIS L 1902:1998 and the Japanese Association for the Functional Evaluation of Textiles, it was concluded that PBT/PANI or PBT- $g$ MA/PANI suppressed the growth of E. coli. Furthermore, we noted that all the samples containing PBT- $g$-MA/PANI exhibited a higher degree of bacterial suppression than the corresponding samples of PBT/PANI, which was attributed to imide formation from the condensation of the anhydride carboxyl groups of PBT-g-MA with the amino groups of PANI. This led to stronger electrostatic interactions in PBT-g-MA/PANI and resulted in greater antibacterial activity; bacterial strains with an extracellular capsule such as E. coli carry less negative charge and are less prone to adsorption by the positively charged surface of PBT-g-MA/PANI.

\section{Conclusions}

The compatibility properties of PBT/PANI composites were improved when PBT-g-MA was used in place of PBT. The composite of PBT- $g$-MA with PANI led to the formation of imide functional groups that were not present in analogous PBT/PANI composites. These imide groups were responsible for different electrical properties between the two copolymers. FTIR spectra and ${ }^{13} \mathrm{C}$ solid-state NMR indicated that the MA moiety had been successfully grafted onto the PBT copolymer and that imide bonds had formed in the PBT-g-MA/PANI composites. The SEM microphotographs indicated that the wettability of PANI in PBT-g-MA was much better than the wettability of PANI in ungrafted PBT. Maximum tensile strengths occurred at about 9 $\mathrm{wt} \%$ PANI, but excess PANI reduced the compatibility of the composite components due to aggregation of PANI. The electrical resistivity of the PBT$g$-MA/PANI (9 wt $\%$ ) composite was $8.1 \cdot 10^{6} \Omega / \mathrm{sq}$, which is $10^{7}$-fold lower than that of neat PBT, affording it high antistatic efficiency. Antibacterial activity was enhanced upon addition of $3 \mathrm{wt} \%$ PANI into PBT- $g$-MA and resulted in high ABI and KBI values. In summary, the current study demonstrates a method for enhancing the compatibility between PBT and PANI and improving the antibacterial and antistatic properties of PBT- and PANIbased composites.

\section{References}

[1] Tourillon G., Garnier F.: Effect of dopant on the physicochemical and electrical properties of organic conducting polymers. The Journal of Physical Chemistry, 87, 2289-2292 (1983).

DOI: $10.1021 / \mathrm{j} 100236 \mathrm{a} 010$

[2] Zhang K., Zhang L. L., Zhao X. S., Wu J.: Graphene/ polyaniline nanofiber composites as supercapacitor electrodes. Chemistry of Materials, 22, 1392-1401 (2010).

DOI: $10.1021 / \mathrm{cm} 902876 \mathrm{u}$

[3] Vercelli B., Zotti G.: Polypyrrole self-assembled monolayers and electrostatically assembled multilayers on gold and platinum electrodes for molecular junctions. Chemistry of Materials, 18, 3754-3763 (2006). DOI: $10.1021 / \mathrm{cm} 060802 \mathrm{e}$

[4] Liu P., Wu Y., Pan H., Ong B. S., Zhu S.: High-performance polythiophene thin-film transistors processed with environmentally benign solvent. Macromolecules, 43, 6368-6373 (2010). DOI: $10.1021 / \mathrm{ma1} 00212 \mathrm{~h}$

[5] Li Y., Wang Z., Wang Q., Wang C., Xue G. A.: A facile and efficient route for coating polyaniline onto positively charged substrate. Macromolecules, 43, 44684471 (2010). DOI: $10.1021 / \mathrm{ma1} 00475 \mathrm{u}$

[6] Fowler J. D., Virji S., Kaner R. B., Weiller B. H.: Hydrogen detection by polyaniline nanofibers on gold and platinum electrodes. The Journal of Physical Chemistry C, 113, 6444-6449 (2009).

DOI: $10.1021 / j p 810500 \mathrm{q}$ 
[7] Li G., Martinez C., Semancik S.: Controlled electrophoretic patterning of polyaniline from a colloidal suspension. Journal of the American Chemical Society, 127, 4903-4909 (2005).

DOI: $10.1021 / \mathrm{ja} 0441763$

[8] Chen Y., Kang E. T., Neoh K. G., Tan K. L.: Surface functionalization of poly(tetrafluoroethylene) films via consecutive graft copolymerization with glycidyl methacrylate and aniline. The Journal of Physical Chemistry B, 104, 9171-9178 (2000).

DOI: $10.1021 / j p 000152 \mathrm{~g}$

[9] Wang P., Tan K. L., Zhang F., Kang E. T., Neoh K. G.: Synthesis and characterization of poly(ethylene glycol)grafted polyaniline. Chemistry of Materials, 13, 581587 (2001).

DOI: $10.1021 / \mathrm{cm} 000829 \mathrm{c}$

[10] Sanchez C., Soler-Illia G. J. de A. A., Ribot F., Lalot T., Mayer C. R., Cabuil V.: Designed hybrid organicinorganic nanocomposites from functional nanobuilding blocks. Chemistry of Materials, 13, 3061-3083 (2001).

DOI: $10.1021 / \mathrm{cm} 011061 \mathrm{e}$

[11] Wu Q., Wang Z., Xue G.: Controlling the structure and morphology of monodisperse polystyrene/polyaniline composite particles. Advanced Functional Materials, 17, 1784-1789 (2007).

DOI: $10.1002 / \mathrm{adfm} .200700170$

[12] Chen C-F., Lee K-H., Chiu W-Y.: Synthesis and characterization of poly(butyl acrylate-methyl methacrylate)/polyaniline core-shell latexes. Journal of Applied Polymer Science, 104, 823-830 (2007).

DOI: $10.1002 / a p p .25624$

[13] Wang Y., Jing X.: Preparation of polystyrene/polyaniline core/shell structured particles and their epoxybased conductive composites. Polymer International, 56, 126-131 (2007).

DOI: $10.1002 /$ pi.2119

[14] Levchik S. V., Weil E. D.: Flame retardancy of thermoplastic polyesters - A review of the recent literature. Polymer International, 54, 11-35 (2005).

DOI: $10.1002 /$ pi.1663

[15] Danès F., Garnier B., Dupuis T., Lerendu P., Nguyen T-P.: Non-uniformity of the filler concentration and of the transverse thermal and electrical conductivities of filled polymer plates. Composites Science and Technology, 65, 945-951 (2005).

DOI: $10.1016 /$ j.compscitech.2004.10.017

[16] Gallo E., Braun U., Schartel B., Russo P., Acierno D.: Halogen-free flame retarded poly(butylene terephthalate) (PBT) using metal oxides/PBT nanocomposites in combination with aluminium phosphinate. Polymer Degradation and Stability, 94, 1245-1253 (2009). DOI: 10.1016/j.polymdegradstab.2009.04.014
[17] Dasaradhudu Y., Narasimha Rao V. V. R.: DC electrical conduction in solution-grown pure and iodinedoped poly(butylene terephthalate) films. Polymer International, 35, 329-335 (1994).

DOI: $10.1002 /$ pi.1994.210350405

[18] Braun U., Bahr H., Sturm H., Schartel B.: Flame retardancy mechanisms of metal phosphinates and metal phosphinates in combination with melamine cyanurate in glass-fiber reinforced poly(1,4-butylene terephthalate): The influence of metal cation. Polymers for Advanced Technologies, 19, 680-692 (2008).

DOI: $10.1002 /$ pat.1147

[19] Jang S. H., Kim Y. H., Lim S., Choi G. D., Kim S. H., Kim W. N.: Effects of fiber characteristics on the mechanical and rheological properties of poly(butylene terephthalate)/glass fiber composites. Journal of Applied Polymer Science, 116, 3005-3012 (2010). DOI: 10.1002/app.31844

[20] Chisholm B. J., Moore R. B., Barber B., Khouri F., Hempstead A., Larsen M., Olson E., Kelley J., Balch G., Caraher J.: Nanocomposites derived from sulfonated poly(butylene terephthalate). Macromolecules, 35, 5508-5516 (2002). DOI: $10.1021 / \mathrm{ma} 012224 \mathrm{n}$

[21] Wu C-S.: Renewable resource-based composites of recycled natural fibers and maleated polylactide bioplastic: Characterization and biodegradability. Polymer Degradation and Stability, 94, 1076-1084 (2009). DOI: $10.1016 /$ j.polymdegradstab.2009.04.002

[22] Balabanovich A. I., Zevaco T. A., Schnabel W.: Fire retardance in poly(butylene terephthalate). The effects of red phosphorus and radiation-induced cross-links. Macromolecular Materials Engineering, 289, 181-190 (2004). DOI: $10.1002 /$ mame.200300153

[23] Vicente A. I., Campos J., Bordado J. M., Ribeiro M. R.: Maleic anhydride modified ethylene-diene copolymers: Synthesis and properties. Reactive and Functional Polymers, 68, 519-526 (2008).

DOI: $10.1016 /$ j.reactfunctpolym.2007.10.026

[24] Zheng W., Levon K., Taka T., Laakso J., Österholm JE.: Phase behaviors and interactions of $N$-alkylated polyanilines and ethylene-co-vinyl acetate blends. Journal of Polymer Science Part B: Polymer Physics, 33, 1289-1306 (1995). DOI: $10.1002 /$ polb.1995.090330814

[25] Rodrigues P. C., Cantão M. P., Janissek P., Scarpa P. C. N., Mathias A. L., Ramos L. P., Gomes M. A. B.: Polyaniline/lignin blends: FTIR, MEV and electrochemical characterization. European Polymer Journal, 38, 22132217 (2002). DOI: $10.1016 / \mathrm{S} 0014-3057(02) 00114-3$ 
[26] Jansen M. A. G., Goossens J. G. P., de Wit G., Bailly C., Koning C. E.: The microstructure of poly(butylene terephthalate) copolymers via ${ }^{13} \mathrm{C}$ NMR sequence distribution analysis: Solid-state copolymerization versus melt copolymerization. Analytica Chimica Acta, 557, 19-30 (2006).

DOI: $10.1016 /$ j.aca.2005.05.063

[27] Im J. S., Kim J. G., Lee S-H., Lee Y-S.: Enhanced adhesion and dispersion of carbon nanotube in PANI/PEO electrospun fibers for shielding effectiveness of electromagnetic interference. Colloids and Surfaces A: Physicochemical and Engineering Aspects, 364, 151-157 (2010).

DOI: $10.1016 /$ j.colsurfa.2010.05.015

[28] De Risi F. R., D’Ilario L., Martinelli A.: Synthesis and characterization of epoxidized polybutadiene/polyaniline graft conducting copolymer. Journal of Polymer Science Part A: Polymer Chemistry, 42, 3082-3090 (2004).

DOI: $\underline{10.1002 / \text { pola.20150 }}$
[29] Chen S. A., Fang W. G.: Electrically conductive polyaniline-poly(vinyl alcohol) composite films: Physical properties and morphological structures. Macromolecules, 24, 1242-1248 (1991).

DOI: $10.1021 / \mathrm{ma} 00006 \mathrm{a} 004$

[30] Wu C-S.: Preparation and characterization of a polycaprolactone $/ \mathrm{C}_{60}$ composite and its improved counterpart (PCL- $\left.\mathrm{NH}_{2} / \mathrm{C}_{60}-\mathrm{OH}\right)$. Journal of Applied Polymer Science, 114, 3489-3499 (2010).

DOI: $10.1002 / a p p .30833$

[31] Blythe A. R.: Electrical resistivity measurements of polymer materials. Polymer Testing, 4, 195-209 (1984).

DOI: 10.1016/0142-9418(84)90012-6

[32] Narkis M., Lidor G., Vaxman A., Zuri L.: New injection moldable electrostatic dissipative (ESD) composites based on very low carbon black loading. Journal of Electrostatics, 47, 201-214 (1999).

DOI: $10.1016 / \mathrm{S} 0304-3886(99) 00041-8$ 